\title{
Improving transition from paediatric to adult cystic fibrosis care: programme implementation and evaluation
}

\author{
Megumi J Okumura, ${ }^{1,2,3}$ Thida Ong, ${ }^{4}$ Diana Dawson, ${ }^{5}$ Dennis Nielson, ${ }^{5}$ \\ Nancy Lewis, ${ }^{5}$ Martha Richards, ${ }^{5}$ Claire D Brindis, ${ }^{3,6}$ \\ Mary Ellen Kleinhenz ${ }^{7}$
}

- Additional material is published online only. To view please visit the journal online (http://dx.doi.org/10.1136/bmjgs2013-002364)

For numbered affiliations see end of article.

\section{Correspondence to} Dr Megumi J Okumura, Department of Pediatrics, University of California, San Francisco, 3333 California Street, STE 245, San Francisco, CA 94118, USA; Okumuram@peds.ucsf.edu

Received 26 July 2013 Revised 30 November 2013 Accepted 3 December 2013

CrossMark

To cite: Okumura MJ, Ong T, Dawson D, et al. BMJ Qual Saf 2014;23:i64-i72.

\begin{abstract}
Background The paradigm of cystic fibrosis (CF) care has changed as effective therapies extend the lives of patients well into adulthood.

Preparing for and maintaining high quality CF care into the adult healthcare setting is critical for prolonged survival. Unfortunately, this transfer process from the paediatric to the adult CF centre is met with a variety of challenges.
\end{abstract}

Objective and methods The objective of this quality improvement (QI) project was to develop, implement and evaluate a theory-based programme for transition from paediatric to adult CF care. In a multi-phase process, the paediatric and adult programmes developed a transition curriculum, addressed care standards and standardised patient transfer protocols. We evaluated the impact of this process through staff surveys, review of field notes from QI meetings, tracking transfers and responses of patients to the Transition Readiness Assessment Questionnaire (TRAQ) at the start of the programme and 18 months after initiation.

Results The collaboration between the paediatric and adult teams continued through quarterly meetings over the past 4 years. This has provided a forum that sustained our transition programme, harmonised care across CF centres and addressed other needs of our CF centre. Discussion of transition with families in the paediatric centre increased twofold (35\% to $73 \% \mathrm{p}<0.001)$, and resulted in a trend towards improved patient TRAQ self-advocacy scores and decreased in-hospital transfer.

Conclusions We successfully created a curriculum and process for transition from paediatric to adult CF care at our centres. This collaboration shapes the communication between our paediatric and adult CF care teams and enables ongoing feedback among patients, families and providers. The impact of our transition programme on long-term patient morbidity will require future evaluation.

\section{BACKGROUND}

Advances in cystic fibrosis (CF) treatment have changed CF from a disease lethal in early childhood to a chronic illness with a median survival of 40 years. ${ }^{1} 2$ Emerging small molecule therapies hold promise to further extend survival. ${ }^{3}$ More than $48 \%$ of CF patients in the USA are age 18 years or older, and this number is increasing. ${ }^{4}$ For young adults with CF, the transition from paediatric to adult care should be a triumphant milestone; however, time of transfer has been associated with morbidities such as loss of pulmonary function, decrease in body mass index (BMI) and hospitalisations. ${ }^{5} 6$ Increased morbidity in young adults with chronic disease is not unique to $\mathrm{CF}$; similar trends occur in youth with other chronic health conditions and reflect challenges of self-care and treatment adherence. $^{7-15}$

Not surprisingly, both paediatric and adult providers face challenges in caring for young adult patients. ${ }^{16}$ Providers in the nationwide network of Cystic Fibrosis Foundation Care Centers work in multidisciplinary teams and deliver expert CF-specific care in paediatric or adult care settings which are often geographically, administratively and professionally separate. Providers struggle to meet the psychosocial challenges and ensure the necessary skills for young adults to be successful adult consumers of healthcare. ${ }^{18-22}$ While multiple reports endorse the standard that 
paediatric providers should prepare their patients for transfer to adult care, there are no generally accepted guidelines to assure smooth transfer from paediatric to adult centres. ${ }^{23-26}$

The University of California, San Francisco Cystic Fibrosis Center (UCSF-CFC) paediatric and adult teams recognised poor coordination in the transfer of paediatric patients to adult care. While some young adult patients transferred to adult care by choice, others were transferred because of being over the age limit of care in the paediatric hospital, marriage, pregnancy or incarceration. Often these transfers were unplanned and occurred during hospitalisation for acute illness, raising a concern for later morbidities. ${ }^{17} 27$ We hypothesised that by jointly formalising a transition process between the two programmes, our providers would improve the care delivered to adolescents and young adults with CF and strengthen young adult self-management and self-advocacy skills. The transition programme was developed around three principles:

1. Transition is a responsibility shared by the paediatric and adult CF teams.

2. Patient introduction to transition in a framework of developmental milestones would promote sufficient knowledge of $\mathrm{CF}$ and skills in self-care to maintain their health and access therapies.

3. Evaluation of the transition programme would allow the CF centre to recognise and sustain positive impacts on patient outcomes and the process of care.

This paper describes the theory supporting our approach to transition, the implementation process, and the effect of the transition programme on the patients, providers and UCSF-CFC.

\section{METHODS}

\section{UCSF-CFC description}

The UCSF-CFC averages an annual census of 160 adult and paediatric patients, with an average of 5-8 patients transferring to the adult centre per year. The paediatric CF clinics are located on the main San Francisco campus and at a larger, suburban outreach clinic 40 miles away. A single separate adult CF clinic is located on the main city campus. Paediatric and adult CF teams admit patients to the same hospital. Paediatric and adult care teams are led by paediatric or adult pulmonary medicine specialists; team members include nurse practitioners, social workers, respiratory therapists and nutritionists. Except for one nurse practitioner trained to see both paediatric and adult patients, there is little overlap of paediatric and adult team members.

\section{UCSF-CFC paediatric to adult transfers prior to transition initiative}

From 2005 to 2008,20 patients transferred from the paediatric to adult centre.
Forty per cent of these patients transferred to adult care during an inpatient hospitalisation without prior contact with the adult CF care team. Eighty per cent of patients transferred required multiple hospitalisations in the year following transfer. During this interval, adult CF providers attempted to facilitate transition by introducing the adult $\mathrm{CF}$ team through outreach services at the suburban paediatric clinic or meeting with families during paediatric hospitalisations. Neither of these interventions solved the poor transfer outcomes.

\section{Identification of barriers to transitioning}

Growing frustration with the transfer process prompted physician leaders of the paediatric and adult CF programmes to perform a centre needs assessment. Care providers identified and reviewed transition barriers for 26 paediatric patients aged 15-26 years. The work generated served as the basis for a quality improvement (QI) proposal funded by the Cystic Fibrosis Foundation (CFF) and was the impetus to formalise a QI transition programme between our paediatric and adult centres.

\section{Development of the transition programme}

Theoretical framework underlying the transition programme

Three theories of change ${ }^{28}$ provided the basis for the design of our transition programme (figure 1) as a single approach to change would not likely address the barriers encountered by the clinic. Barriers identified in the needs assessment were approached from an ecological model. This model acknowledges that overcoming transition barriers must be approached in a multi-level process: intrapersonal (within patient and provider), interpersonal (among providers and between providers and patients), and within the community (clinic/hospital). The specific interventions to address barriers per level are listed in online supplementary appendix 1 . Social Cognitive Theory of Change provided the framework for changes in provider and patient interaction through reciprocal determinism (changing the environment through educational materials used by providers), behavioural capacity (ensuring skills were met), expectations (improving discussion on health outcomes), selfefficacy (curriculum to improve understanding and disease), modelling (through repetition and teaching) and reinforcements (achieved by reviewing set goals with providers). Educational transition materials for patients and families were developed within the Health Belief Model framework (table 1). Materials focused on addressing gaps in knowledge of disease and the perceived beliefs of health status. The goals of the materials were to develop patient self-management and self-advocacy skills while documenting their readiness to engage in adult responsibilities related to their CF care. 


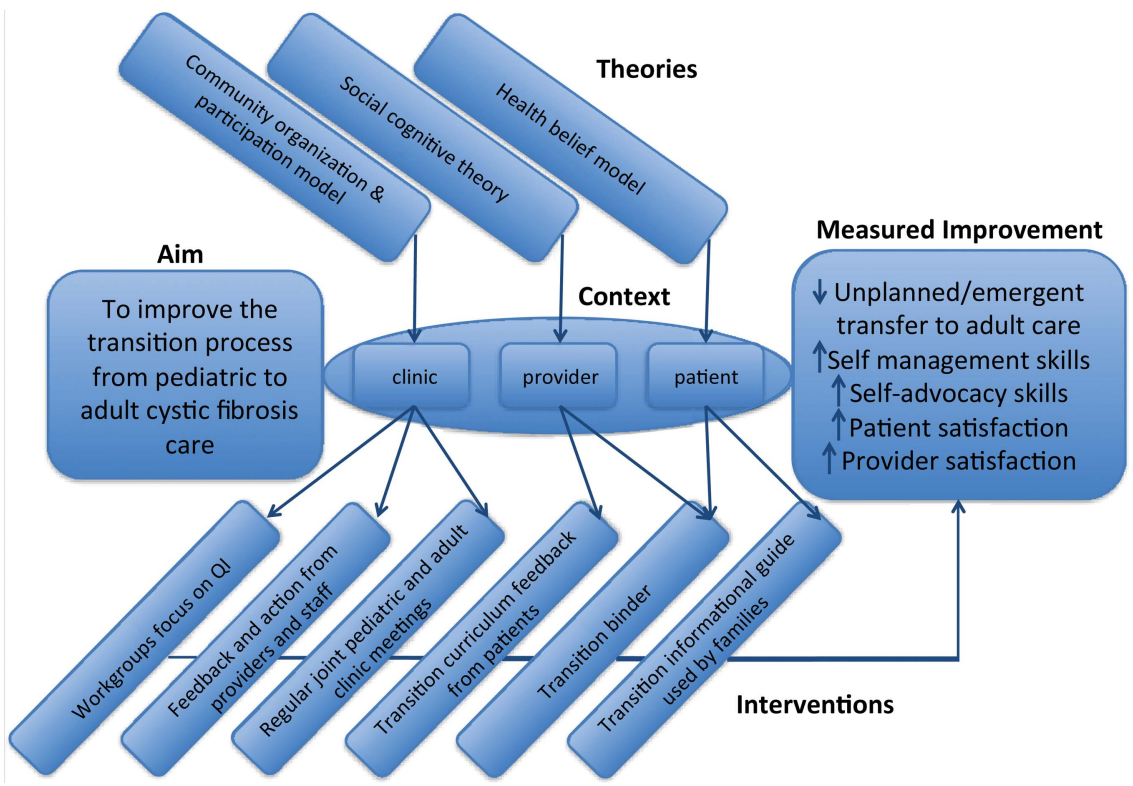

Figure 1 Driver diagram for cystic fibrosis programme.

Provider and clinic/hospital barriers: creating a culture of shared responsibility

To facilitate communication between the paediatric and adult centres, joint $3 \mathrm{~h}$ meetings were held every 2 months with published, time-allotted agendas to optimise productivity. Meetings were attended by respiratory therapists, social workers, pharmacists, dieticians and the clinical providers. At least one representative from each of the three clinic sites attended each meeting. During these meetings, staff addressed deficiencies in the programme and presented ideas and options for approaching the issue at hand. The physician-lead then assigned a team member to assemble a work group to develop a clinical solution to the problem. For example, annually, a QI meeting was devoted to transition readiness assessments of patients 16 years and older. To facilitate these patient reviews, the adult team social worker agreed to work with peers from the paediatric clinics to develop the Transition Readiness Tool. At the annual patient

Table 1 Health Belief Model: contents of the transition notebook

Domains
Perceived susceptibility
microbiologic profile and its effects on long term health

BMI, body mass index; CF, cystic fibrosis; CFF, Cystic Fibrosis Foundation. 


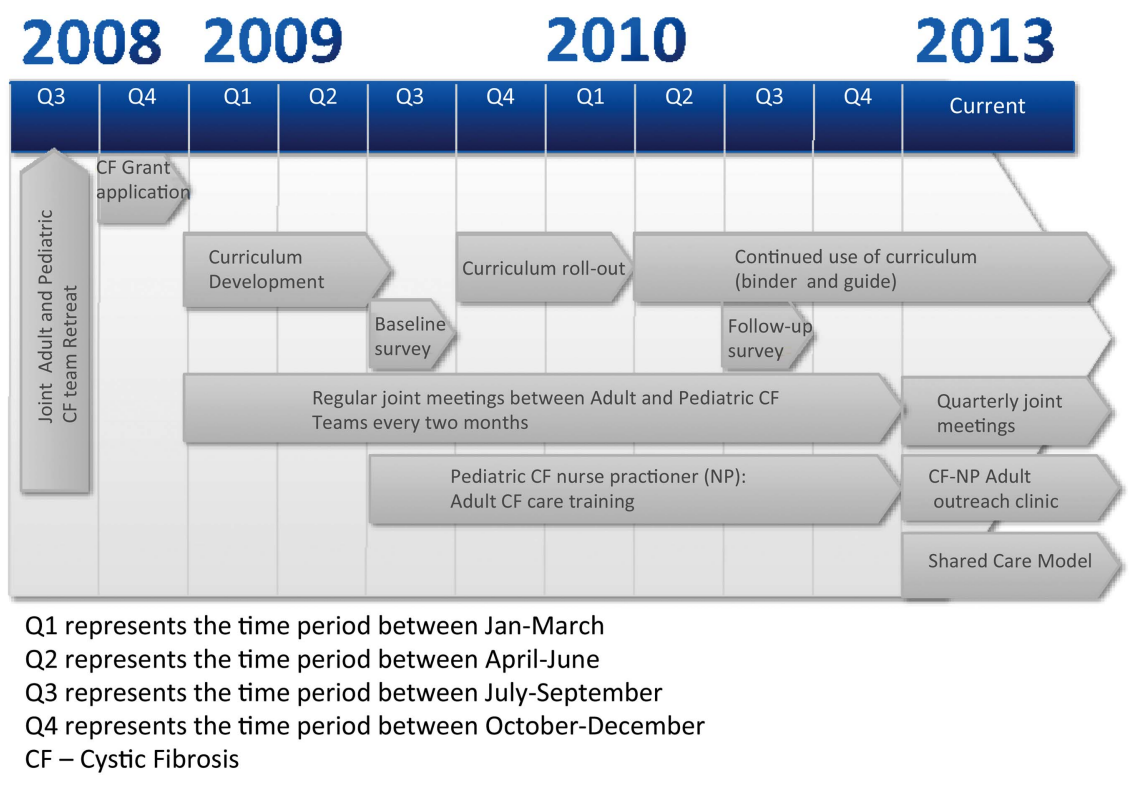

Figure 2 Programme timeline.

review, adult and paediatric providers worked from this template to discuss and record challenges in care (eg, psychosocial, developmental) that could persist following transfer. Nutritionists from the paediatric and adult clinics observed that the approach to patients with low BMI varied among the clinic sites and clinicians. Working from the published nutrition guidelines of the CFF, the nutritionists developed a Nutrition Map to standardise nutritional assessments and interventions in our adult and paediatric clinics. A timeline illustrating the programme and dynamics of the QI processes is shown in figure 2.

\section{Patient/family barriers: developing and implementing transition materials}

We developed transition materials to educate patients, communicate transition goals and convey our centre's approach to this process. ${ }^{18} 29$ The materials, a transition guide and notebook were developed in a series of rapid Plan-Do-Study-Act (PDSA) ${ }^{30}$ cycles over the 6-month process. Initially, a work group comprised of physicians and nurses created an educational guide entitled 'Transitions'. The transition guide emerged as an action item between QI meetings as team members felt the need for tools to teach patients and families about CF care (Plan). The guide amalgamated transition materials from various chronic disease clinics, prior work of other CF centres and industry adherence tools. Our guide introduces transition in an age-based framework of developmental milestones. CF knowledge and skills are organised by age, in 2-3-year increments, as checklists delineating the transition curriculum. Each checklist incorporates discussion points for therapies, patient specific details of health status such as pulmonary function testing, and challenges of maturation including peer pressure, sexuality and work/education goals. The guide focuses on knowledge of CF, emotional coping, adherence strategies, and increasing autonomy in self-care within healthcare visits consistent with the Social Cognitive Theory Model and the Health Belief Model (figure 1 and table 1).

While our guide provided information, it did not support active learning. A pulmonary fellow developed a working notebook (Plan) that could be personalised to each patient, fostered interaction with the care team, and recorded challenges and progress through each of the 2-year blocks. The transition notebook was shaped by input from providers and CF team members (Do). The tool was then pretested on patients and families, vetting content, format and language, as well as research into other tools (Study). Information of the pre-testing was then incorporated into the tool and presented for group discussion at a subsequent QI meeting and approval by clinic members for implementation or change in the clinic (Act). The PDSA cycles continue as the supplemental resources and tools are added to the notebook materials with editing for relevance and to meet clinical and patient need. The guides and notebooks are given to all patients 8 years and older as standard of care. Patients are encouraged to bring the notebook to each clinic visit to discuss transition topics. If notebooks are not brought to clinic, patients receive a copy of the age-appropriate checklist excerpted from the guide. Provider documentation includes short-term interval goals to keep track of patient curriculum progress. Production costs for the guide and notebook were funded by the CFF Quality grant and an internal grant for pilot patient care projects. The approximate cost for patient materials was $\$ 8$ per patient ( $\$ 3$ per colour guide and $\$ 5$ for notebook) in 2009 . 
Evaluation of the transition programme

Transition programme evaluation

Provider feedback following implementation assessed length of time and barriers to implementation during bimonthly and then quarterly meetings as part of the PDSA cycles. To measure the perceptions of the intervention on patients and families and its effect on transition outcomes, a survey was administered in the paediatric CF clinic at the start of the QI intervention and 18 months after the rollout process. The survey included closed questions on demographics and the transition materials (usefulness of guide and notebook, actual use of notebook and guide, which specific notebook components were used in clinic and at home). We also elicited open-ended feedback on the transition programme. Families of patients aged 8 and older, and patients aged 16 and older in the paediatric centre were given the survey just prior to the transition programme (Time 1) and 18 months after complete rollout of the programme (Time 2).

Transition readiness evaluation of patients

To evaluate patient transition readiness, we used the Transition Readiness Assessment Questionnaire (TRAQ). ${ }^{31}$ This validated 29 -item transition readiness tool measures two major domains: 'skills for chronic condition self-management' and 'skills for selfadvocacy and communication with health providers'. Each item of TRAQ is scored on a $1-5$ point scale, where 1 is the lowest skill level. An average score was generated for each domain. TRAQ assessments were performed on paediatric CF patients 16 and older at Time 1 and Time 2. Adult centre patients aged 18-25 were approached to complete the TRAQ assessment at Time 1 to serve as a comparison group.

\section{Patient transfer status}

A retrospective chart review assessed the ways patients transferred from the paediatric to adult clinic before and after the transition programme started. In addition we evaluated differences in BMI and hospitalisations 1 year after transfer to the adult centre. The first interval extended from March 2005 to July 2008, which preceded the start of the transition programme activities and serves as a historical baseline. The second interval extended from October 2009, which was the start of the full transition programme through December 2012.

Ethical consideration

No incentive was given to the patients or families to complete surveys. The University of California Committee on Human Subjects approved the QI project and evaluation.

\section{Analysis plan}

Demographic and clinical data from the CFF National Patient Registry was accessed to identify eligible patients in the adult and paediatric CF centres. The Wilcoxon matched-pairs signed-ranks test was used to determine difference between paired Time 1 and Time 2 TRAQ scores. The Wilcoxon rank-sum, $\chi^{2}$ and Fisher's exact tests were used to compare non-paired continuous and categorical variables accordingly. STATA V.11 was used for all analyses.

\section{RESULTS}

Paediatric and adult providers sharing the responsibility of transition: quality and process feedback

Online supplementary appendix 1 outlines the barriers identified by providers, interventions intended to mitigate these challenges and comments on the ongoing improvement process at the initial joint meeting in August 2008. The paediatric and adult CF team assembled as a group every 2 months for over 2 years to review progress, critique materials, and plan implementation and evaluation. Although travel compensation ended in 2010, the joint paediatric and adult QI meetings continue on a quarterly basis as the routine culture of our CF care centre. These meetings are essential to sustain the transfer of patients and are the forum for promoting improvement in overall CF care delivery at our centre. Ad hoc work groups, such as the paediatric and adult nutritionists, spearheaded standardisation of nutrition goals and laid the groundwork for future protocol standardisation at UCSF-CFC through active PDSA cycles. Transition initiative funding provided partial support of one paediatric nurse practitioner, enabling her to obtain training in adult CF care. Now, as a provider trained in paediatric and adult CF care, she shares time at the paediatric suburban CF clinic to provide adult CF care for youth who are challenged by schedules or distance. With the introduction of a hospital-wide outpatient electronic medical record (EMR), access to centre wide documentation of CF clinical care is available to all providers.

\section{Provider, youth and parent feedback of programme and materials}

Providers reported an average of $30 \mathrm{~min}$ introducing the transition programme and materials. Acute illness visits were not used to address the transition curriculum. The age-based curriculum in increments of 23 years allowed a flexible pace to review aspects of transition readiness.

At Time 1, 56 of 92 (61\%) eligible patients or their parents responded to the survey. At Time 2, families reporting the discussion of transition with paediatric providers increased from $35 \%$ to $73 \% \quad(p<0.01)$. Table 2 summarises the use and usefulness of the notebook and guide to parents and patients. The personal notebook was preferred over the overview guide (table 2). Families used the notebook predominantly in clinic with their child's providers versus at home (70\% vs 40\%). Youth mirrored this pattern of preferential clinic versus home use (68\% vs 50\%). The most utilised sections were: short term goal (to-do) list 
Table 2 Views of transition materials

\begin{tabular}{lll}
\hline & $\begin{array}{l}\text { Family } \\
\mathrm{N}=23(\%)\end{array}$ & $\begin{array}{l}\text { Young adults } \\
\mathrm{N}=22(\%)\end{array}$ \\
\hline Used the transition guide over the past year & 45 & 47 \\
Found the guide useful & 69 & 42 \\
Used the transition notebook in the past & 86 & 81 \\
year & & \\
Found the notebook useful & 95 & 68 \\
\hline
\end{tabular}

(68\%), personalised CF registry graphs of lung function and BMI over time (port CF graphs) (43\%), and informational handouts (30\%).

Comments provided in the open-ended feedback portion in the survey emphasised that the transitions materials and overall project were well received. '[This transition program has] empowered [my] daughter to take responsibility for her care!' Feedback also highlighted barriers to $\mathrm{CF}$ care that had not been addressed in initial implementation: 'Appointments at UCSF have become all day events; it is difficult for young college students to get time off from school and commute all the way to [San Francisco].'

\section{Transition readiness}

Eighteen paediatric patients completed the postintervention survey, with eight patients completing both the baseline survey and the post-intervention survey. This discrepancy reflected patients aging into the intervention cohort during the study period, and differential non-response. TRAQ self-advocacy scores at Time 2 vs Time 1 were improved to scores similar to their adult centre peers, but were not statistically significant (paired: 3.8 vs $4.8, p=0.058$, table 3 ). There was no significant difference in the TRAQ self- management domain between Time 1 and Time 2 (table 3). Compared to baseline adult programme patients, paediatric patients scored significantly lower on self-management skills ( 4.0 vs $4.5 \mathrm{p}<0.05)$, but their self-advocacy skills were comparable their adult centre peers.

\section{Transfer outcomes}

Based on chart review, nine young adults transitioned to the adult programme during this pilot project (from October 2009 to December 2012). We compared these nine transfers to the 20 patients who transferred to the adult programme between March 2005 and July 2008. Four patients transferred readily to adult care with scheduled outpatient visits. During the first year of the transition programme two patients transferred to adult care during urgent hospitalisation without preparation of patient/family or adult providers for transfer of care. This undesirable outcome decreased from 8/20 (40\%) pre-transition programme to $2 / 9 \quad(22 \%) \quad(p=0.43) \quad$ post-transition. Since September 2010, there have been no urgent hospital transfers to adult care. Three patients required more gradual transition of care to the adult CF programme due to initial refusal by patient or parent. To address their unique transition needs, these patients accessed CF care through the paediatric or adult teams through a 'shared care model' continued over a number of months until patient and family were comfortable and settled in adult care. There was a trend towards fewer hospitalisations 1 year post-transfer, with the rate decreasing from $80 \%(16 / 20)$ to $44 \%(4 / 9)(p=0.09)$ after the transition programme was initiated. There was no change in BMI status.

Table 3 Transition readiness assessment questionnaire (TRAQ) results

\begin{tabular}{|c|c|c|c|c|c|}
\hline & \multirow{3}{*}{$\begin{array}{l}\text { Adult centre (18-25) } \\
\text { Mean TRAQ score } \\
\text { (CI) baseline* } \\
\text { Pre-intervention } \\
\text { (Time 1) }\end{array}$} & \multicolumn{2}{|c|}{ Unpaired youth paediatric clinic } & \multicolumn{2}{|c|}{ Paired youth paediatric clinic } \\
\hline & & \multicolumn{2}{|c|}{ Mean TRAQ score $(\mathrm{Cl}) \dagger$} & \multicolumn{2}{|c|}{ Mean TRAQ score $(\mathrm{CI}) \ddagger$} \\
\hline & & $\begin{array}{l}\text { Pre-intervention } \\
\text { (Time 1) }\end{array}$ & $\begin{array}{l}\text { Post-intervention } \\
\text { (Time 2) }\end{array}$ & $\begin{array}{l}\text { Pre-intervention } \\
\text { (Time 1) }\end{array}$ & $\begin{array}{l}\text { Post-intervention } \\
\text { (Time 2) }\end{array}$ \\
\hline & $n=10$ & $\mathrm{n}=11$ & $\mathrm{n}=18$ & $\mathrm{n}=8$ & $\mathrm{n}=8$ \\
\hline $\begin{array}{l}\text { 1. Skills for chronic condition } \\
\text { self-management }\end{array}$ & $4.5 \S(3.9$ to 5.0$)$ & $3.7(2.9$ to 4.6$)$ & $3.4(2.9$ to 3.9$)$ & $3.4(2.3$ to 4.4$)$ & $4.0(3.5$ to 4.6$)$ \\
\hline $\begin{array}{l}\text { 2. Self-advocacy and } \\
\text { communication with } \\
\text { health providers }\end{array}$ & $4.7(4.3$ to 5.0$)$ & $4.0(3.4$ to 4.7$)$ & $4.6(4.4$ to 4.8$)$ & $3.9(2.8$ to 4.9$)$ & $4.8(4.6$ to 4.9$)$ \\
\hline \multicolumn{6}{|c|}{$\begin{array}{l}\text { Each item of TRAQ is scored on a 1-5 point scale, where } 1 \text { is the lowest skill level, for example, 'No, I do not know how but I want to learn'; and } 5 \text {, the } \\
\text { highest, with the response, 'Yes, I always do this when I need to'. Each item was used to generate a composite average of the two domains, where the } \\
\text { lower the score, the less likely the youth is prepared to transition. } \\
\text { * } p \text { values generated from the Wilcoxon rank-sum test comparison of baseline adults <age } 25 \text { in adult CF centre versus Time } 2 \text { of all paediatric survey } \\
\text { participants in the paediatric centre. } \\
\text { tp values generated from the Wilcoxon rank-sum test comparing Time } 1 \text { versus Time } 2 \text { of unpaired paediatric patients (comparing respondent groups in } \\
\text { pre- and post-, but not within patient comparison). } \\
\neq p \text { values generated from the Wilcoxon matched-pairs signed-ranks test comparing Time } 1 \text { versus Time } 2 \text { of paired (paired within individual) paediatric } \\
\text { patients. } \\
\S p<0.05 \text {. }\end{array}$} \\
\hline
\end{tabular}




\section{DISCUSSION}

Over 2 years, we developed and implemented a programme to organise transition of CF patients from paediatric to adult care. The successful implementation was based on approaching an intervention through an ecological framework: working on interpersonal, intrapersonal and community (clinic/hospital) levels. Our process supports a culture of shared responsibility for transition among paediatric and adult CF providers, patients and families. Our providers worked to accommodate the needs of patients and families, mitigated worry about separation from familiar paediatric providers and created a costeffective approach to a geographic need for adult CF care in the suburban clinic. As adult providers were integrated into the continuum of care, they acquired sensitivity to the dynamics of chronic illness management through adolescence. Paediatric and adult providers continue collaboration through quarterly meetings to address new challenges and align CF care in the clinics and hospital.

While physician champions provided the opportunity to address the transition process, the team members (nurses, social workers, nutritionists, respiratory therapists, clinic staff) generated the materials and workflows that now support patients, families and clinicians in preparing youth to maintain high quality CF care in adulthood. Components of curriculum and process reflect the efforts and expertise of disciplines and individuals from our care teams. For example, nutritionists, not physicians, spearheaded development of the nutrition education and more rigorous, guideline-based assessments. By working with all levels in the clinic, the staff devised 'miniinterventions' to address each of the barriers found in our transition needs assessment. With inherent buy-in created by responsibility for assessing and improving process, these solutions were readily incorporated and are sustained in the clinic. Other clinics developing their own transition programmes will differ in the specificity of 'mini-interventions' needed to address barriers in transition in their own institution. It is our view that similar issues (eg, communication between systems, self-efficacy, self-management) will challenge transitioning CF patients as they age out of paediatric healthcare.

The transition initiative process identified actionable barriers to transfer. When the adult centre was challenged by a subset of youth who were not prepared to transition by the anticipated timeline, we accepted a paediatric and adult shared model of care to accommodate this group. Increased paediatric and adult collaboration halved the number of unplanned urgent in-hospital transfers.

Our transition curriculum actively engaged patients and families in discussion of transition and jumpstarted the dialogue of key issues in chronic disease management in our paediatric clinics. There was not a significant improvement in self-management scores; curriculum updates and further assessment will be required to evaluate whether we can improve these metrics. Self-advocacy scores, including understanding access to therapies, increased to levels to similar to those observed in transitioned adults, suggesting that stepwise discussion of responsibilities of care is important preparation in the management of chronic disease. Overall, our materials were well received; parents and youth preferred materials that actively engaged them with their providers in addressing the challenges of CF care over passive 'how to' materials. It is likely that transition strategies that attempt to simply 'inform' will not be as useful in helping youth transition. A critical part of this process was regular patient-provider engagement to review transition materials and goals.

We acknowledge the following features to successful implementation of our programme: (1) The grant from the CFF funded staff attendance at frequent, regular meetings between adult and paediatric programmes and allowed the adult CF programme to hire and train a paediatric nurse practitioner as a part-time mid-level provider. (2) Our multidisciplinary group of invested $\mathrm{CF}$ team members was enriched by an adolescentfocused health services researcher who planned surveys, organised the multi-layered analysis of our programme and introduced the concept of theories of change. (3) Implementation of an EMR system throughout the Medical Center facilitated communication among adult and paediatric providers and clinics. (4) Having an accessible clinician common to both the paediatric and adult CF teams allayed familial concern about continuity and access during a process that challenged patients, their families and providers.

We believe the major key to successful development of our programme was working on an ecological framework: recognising the patient/provider and system level factors in transition through the inclusion of families, providers and staff of the clinic. This framework allowed for programme sustainability. The transition process requires shared responsibility of all parties that involves identification of needs and actionable items with systematic follow-up to address and act upon the domains in an ecologic framework. Therefore, we feel that this framework can be replicated in any clinical environment that wishes to address healthcare transitions in their programme.

\section{LIMITATIONS}

Surveys are based on self-report, and though clinical outcomes were monitored, perceptions of quality of care may not reflect actual quality of care received. We had a small sample size, which likely impacted the statistical significance of our metrics, which trended towards improvement but were not statistically significant. Impact on long-term morbidity will need subsequent monitoring. Experiences and data described in 
this paper are based on experiences from a single academic medical CF centre, and therefore other centres will need to consider their particular context.

\section{CONCLUSION}

We successfully developed and implemented a transition programme, which created a culture of shared responsibility between adult and paediatric programmes. The transition programme created mechanisms to identify and address barriers within our transfer process impacting our hospital and clinics, adult and paediatric providers, and patients and families. Working with behavioural theories to improve care delivery, we improved communication and improved the transfer process for families, staff and providers. The collaborative effort between our adult and paediatric programmes continues in regular communication and efforts have expanded to standardise CF care protocols. A change in culture has occurred with paediatric and adult programmes respecting our common interest and acknowledging a shared responsibility of transition. This process of shared responsibility and QI can be implemented in any clinic or service that requires a coordinated transition and eventual transfer of care from paediatric to adult services.

\footnotetext{
Author affiliations

${ }^{1}$ Division of General Pediatrics, University of California, San Francisco, California, USA

${ }^{2}$ Division of General Internal Medicine, University of California, San Francisco, California, USA

${ }^{3}$ Phillip R. Lee Institute for Health Policy Studies, University of California, San Francisco, California, USA

${ }^{4}$ Division of Pediatric Pulmonology, Department of Pediatrics, Seattle Children's Hospital, University of Washington, Seattle, Washington, USA

${ }^{5}$ Division of Pediatric Pulmonology, University of California, San Francisco, California, USA

${ }^{6}$ Division of Adolescent Medicine, University of California, San Francisco, California, USA

${ }^{7}$ Division of Pulmonary, Critical Care, Allergy-Immunology and Sleep Medicine, Department of Internal Medicine, University of California, San Francisco, California, USA

Contributors MO, MEK: conception and design, acquisition of data, analysis and interpretation of data, drafting the article, revising it critically for important intellectual content, final approval of the version to be published. TO, CDB: conception and design, interpretation of data, drafting the article, revising it critically for important intellectual content, final approval of the version to be published. DD, DN, NL, MER: conception and design, revising it critically for important intellectual content, final approval of the version to be published.
}

Funding The paper was supported in part by grants from the Cystic Fibrosis Foundation, U45MC 00002 and U45MC 00023 of the Maternal and Child Health Bureau, Health Services and Resources Administration, US Department of Health and Human Services (CB) and the Agency for Healthcare Research and Quality K08HS017716-01 (MO).

\section{Competing interests None.}

Ethics approval University of California-San Francisco Committee on Human Subjects.

Provenance and peer review Not commissioned; externally peer reviewed.

Data sharing statement Data on views of CF care and views of quality improvement are available from MO.

\section{REFERENCES}

1 Elborn JS, Shale DJ, Britton JR. Cystic fibrosis: current survival and population estimates to the year 2000. Thorax 1991;46:881-5.

2 Hodson ME, Simmonds NJ, Warwick WJ, et al. An international/multicentre report on patients with cystic fibrosis (CF) over the age of 40 years. J Cyst Fibros 2008;7:537-42.

3 Davis PB. Therapy for cystic fibrosis-the end of the beginning? N Engl J Med 2011;365:1734-5.

4 Cystic Fibrosis Foundation Program Specific Patient Registry Report. 2011.

5 Dugueperoux I, Tamalet A, Sermet-Gaudelus I, et al. Clinical changes of patients with cystic fibrosis during transition from pediatric to adult care. J Adolesc Health 2008;43:459-65.

6 Champion EA, Dellon EP. Comparison of clinical changes before and after transition to adult care in patients with cystic fibrosis. American Thoracic Society International Conference. Denver, CO: American Thoracic Society, 2011.

7 Baines JM. Promoting better care: transition from child to adult services. Nurs Stand 2009;23:35-40.

8 Okumura MJ, Heisler M, Davis MM, et al. Comfort of general internists and general pediatricians in providing care for young adults with chronic illnesses of childhood. J Gen Intern Med 2008;23:1621-7.

9 McLaughlin SE, Diener-West M, Indurkhya A, et al. Improving transition from pediatric to adult cystic fibrosis care: lessons from a national survey of current practices. Pediatrics 2008;121:e1160-6.

10 Hewer SC, Tyrrell J. Cystic fibrosis and the transition to adult health services. Arch Dis Child 2008;93:817-21.

11 Rehm RS. A life skills intervention improved psychosocial adjustment in children with cystic fibrosis. Evid Based Nurs 2007;10:47.

12 Parker HW. Transition and transfer of patients who have cystic fibrosis to adult care. Clin Chest Med 2007;28:423-32.

13 Dobbin CJ, Bye PT. Adults with cystic fibrosis: meeting the challenge! Intern Med J 2003;33:593-7.

14 Sawyer SM, Drew S, Yeo MS, et al. Adolescents with a chronic condition: challenges living, challenges treating. Lancet 2007;369:1481-9.

15 Flume PA. Smoothing the transition from pediatric to adult care: lessons learned. Curr Opin Pulm Med 2009;15:611-14.

16 Flume PA, Anderson DL, Hardy KK, et al. Transition programs in cystic fibrosis centers: perceptions of pediatric and adult program directors. Pediatr Pulmonol 2001;31:443-50.

17 Flume PA, Taylor LA, Anderson DL, et al. Transition programs in cystic fibrosis centers: perceptions of team members. Pediatr Pulmonol 2004;37:4-7.

18 Crowley R, Wolfe I, Lock K, et al. Improving the transition between paediatric and adult healthcare: a systematic review. Arch Dis Child 2011;96:548-53.

19 Tuchman LK, Schwartz LA, Sawicki GS, et al. Cystic fibrosis and transition to adult medical care. Pediatrics 2010;125:566-73.

20 Taylor L, Tsang A, Drabble A. Transition of transplant patients with cystic fibrosis to adult care: today's challenges. Prog Transplant 2006;16:329-34; quiz 335.

21 Tuchman LK, Slap GB, Britto MT. Transition to adult care: experiences and expectations of adolescents with a chronic illness. Child Care Health Dev 2008;34:557-63.

22 Zack J, Jacobs CP, Keenan PM, et al. Perspectives of patients with cystic fibrosis on preventive counseling and transition to adult care. Pediatr Pulmonol 2003;36:376-83. 
23 Towns SJ, Bell SC. Transition of adolescents with cystic fibrosis from paediatric to adult care. Clin Respir J 2011;5:64-75.

24 A consensus statement on health care transitions for young adults with special health care needs. Pediatrics 2002;110(6 Pt 2):1304-6.

25 Cooley WC, Sagerman PJ. Supporting the health care transition from adolescence to adulthood in the medical home. Pediatrics 2011;128:182-200.

26 Yankaskas JR, Marshall BC, Sufian B, et al. Cystic fibrosis adult care: consensus conference report. Chest 2004;125(1 Suppl): 1S-39S.

27 Chaudhry SR, Keaton M, Nasr SZ. Evaluation of a cystic fibrosis transition program from pediatric to adult care. Pediatr Pulmonol 2013;48:658-65.
28 National Cancer Institute, U.S. Department of Health and Human Services, National Institutes of Health. Theory at a glance: application to health promotion and health behavior. 2nd edn. National Cancer Institute, 2005. http://www.cancer.gov/ cancertopics/cancerlibrary/theory.pdf (accessed 6 Feb 2013).

29 Center TNHCT. Six Core Elements of Health Care Transition. 2011.

30 Langley GJ. The improvement guide: a practical approach to enhancing organizational performance. 2nd edn. San Francisco: Jossey-Bass, 2009.

31 Sawicki GS, Lukens-Bull K, Yin X, et al. Measuring the transition readiness of youth with special healthcare needs: validation of the TRAQ_-Transition Readiness Assessment Questionnaire. J Pediatr Psychol 2011;36:160-71. 